\title{
Thermodynamical Behaviour of Inhomogeneous Universe with Varying $\Lambda$ in Presence of Electromagnetic Field
}

\author{
Anil Kumar Yadav \\ Department of Physics, Anand Engineering College,Keetham, Agra-282 00\%, \\ India \\ E-mail: abanilyadav@yahoo.co.in, anilyadav.physics@gmail.com
}

\begin{abstract}
Thermodynamical Behaviour of Inhomogeneous Universe with Varying $\Lambda$ in Presence of Electromagnetic Field is obtained. $F_{12}$ is the non-vanishing component of electromagnetic field tensor. To get a deterministic solution, it is assumed that the free gravitational field is Petrov type-II non-degenerate.The value of cosmological constant is found to be small and pasitive supported by recent results from the supernovae observations recently obtained by High-Z Supernovae Ia Team and Supernovae Cosmological Project. A relation between cosmological constant and thermodynamical quantities is established. Some physical and geometric properties of the model are also discussed.
\end{abstract}

Key words : Cosmology, Electromagnetic field, Inhomogeneous universe . PACS: 98.80.Jk, 98.80.-k

\section{Introduction}

A lesson given by the history of cosmology is that the concept of the cosmological term revives in the days of crisis and we have more reasons than ever to belive that the cosmological term is the necessary ingredient of any cosmological model.The possibility of adding a cosmological vacuum energy density to the Einstein field equations raises the question empirical justification of such a step. A positive cosmological constant helps overcome the age problem, conected on the one side with the high estimates of the Hubble parameters and with the age of the globular clusters on the other. Further, it seems that in order to retain the cold dark matter theory in the spacially flat universe most of the critical density should be provided by a passitive cosmological constant [1, 2]. Observationnal data indicate that the cosmologial constant, if nonzero, is smaller than $10^{-55} \mathrm{~cm}^{-} 2$. However, since everything that contributes to the vacuum energy acts as a cosmological constant it can not just be dropped without serious considerations. Moreover particle physics expectations for $\Lambda$ exceeds its present 
value by the factor of order $10^{120}$ ie in a sharp contrast to observations. To explain this apparent discrepancy the point of view has been adopted which allow the $\Lambda$ term to vary in time [3]-[11]. The idea of that during the evolution of universe the energy density of the vacuum decays into the particles thus leading to the decrease of the cosmological constant. As the result one has the creation of particles although the typical rate of the creation is very small.

The creation of matter and entropy from vacuum has been studied via quantum field theory in curved spacetime [12, 13. Most cosmological models exhibit a singurarity which presents difficulties for interpreting quantum effects, because all macroscopic parameters of created particles are infinite there.This leads to the problem of the initial vacuum. A regular vacuum for species of the created particles can be defined in simple terms as a state where all mean values describing the particles, such as energy density, number density, entropy etc are zero. But this simple condition is not achieved in many scenarios, so that either one has to postulate an initial state beyond the singularity, or to assume that there was a non-zero number of particles at the initial vacuum. One attempt to overcome these problems is via incorporating the effect of particle creation into Einstein's field equactions. In the present study I interpret the source of created particles as a decaying vacuum, described phenomenologically by a time-dependent cosmological constant $\Lambda(t)$.

There are significant observational evidence for the detection of Einsteins cosmological constant, $\Lambda$ or a component of material content of the universe that varies slowly with time and space to act like $\Lambda$. Some of the recent discussions on the cosmological constant problem and on cosmology with a time-varying cosmological constant by Ratra and Peebles [14, and Sahni and Starobinsky [15], point out that in the absence of any interaction with matter or radiation, the cosmological constant remains a constant.However, in the presence of interactions with matter or radiation, a solution of Einstein equations and the assumed equation of covariant conservation of stress-energy with a time-varying $\Lambda$ can be found. This entails that energy has to be conserved by a decrease in the energy density of the vacuum component followed by a corresponding increase in the energy density of matter or radiation (see also Carroll, Press and Turner [16], Peebles [17, Padmanabhan [18]). There is a plethora of astrophysical evidence today, from supernovae measurements (Perlmutter et al. [19, Riess et al. 20, Garnavich et al. 21, Schmidt et al. 22, Blakeslee et al. 23], Astier et al. 24]), the spectrum of fluctuations in the Cosmic Microwave Background (CMB) 25], baryon oscillations [26 and other astrophysical data, indicating that the expansion of the universe is currently accelerating. The energy budget of the universe seems to be dominated at the present epoch by a mysterious dark energy component, but the precise nature of this energy is still unknown. Many theoretical models provide possible explanations for the dark energy, ranging from a cosmological term [27] to super-horizon perturbations [28] and timevarying quintessence scenarios [29]. These recent observations strongly favour a significant and a positive value of $\Lambda$ with magnitude $\Lambda\left(\frac{G \hbar}{c^{3}}\right) \approx 10^{-123}$.

The standard Friedman-Robertson-Walker (FRW) cosmological model prescribes a homogeneous and an isotropic distribution for its matter in the de- 
scription of the present state of the universe. At the present state of evolution, the universe is spherically symmetric and the matter distribution in the universe is on the whole isotropic and homogeneous. But in early stages of evolution, it could have not had such a smoothed picture. Close to the big bang singularity, neither the assumption of spherical symmetry nor that of isotropy can be strictly valid. So we consider plane-symmetric, which is less restrictive than spherical symmetry and can provide an avenue to study inhomogeneities. Inhomogeneous cosmological models play an important role in understanding some essential features of the universe such as the formation of galaxies during the early stages of evolution and process of homogenization. The early attempts at the construction of such models have done by Tolman [30] and Bondi [31] who considered spherically symmetric models. Inhomogeneous plane-symmetric models were considered by Taub [32, 33] and later by Tomimura 34, Szekeres 35. Recently, Senovilla 36] obtained a new class of exact solutions of Einstein's equation without big bang singularity, representing a cylindrically symmetric, inhomogeneous cosmological model filled with perfect fluid which is smooth and regular everywhere satisfying energy and causality conditions. Later, Ruis and Senovilla 37] have separated out a fairly large class of singularity free models through a comprehensive study of general cylindrically symmetric metric with separable function of $r$ and $t$ as metric coefficients. Dadhich et al. 38] have established a link between the FRW model and the singularity free family by deducing the latter through a natural and simple in-homogenization and anisotropization of the former. Recently Bali and Tyagi 39], Pradhan et al[40] obtained a plane-symmetric inhomogeneous cosmological models of perfect fluid distribution with electro-magnetic field.

The occurrence of magnetic fields on galactic scale is well-established fact today, and their importance for a variety of astrophysical phenomena is generally acknowledged as pointed out by Zeldovich et al. 41. Also Harrison 42, has suggested that magnetic field could have a cosmological origin. As a natural consequences, we should include magnetic fields in the energy-momentum tensor of the early universe. The choice of anisotropic cosmological models in Einstein system of field equations leads to the cosmological models more general than Robertson-Walker model [43. Strong magnetic fields can be created due to adiabatic compression in clusters of galaxies. Primordial asymmetry of particle (say electron) over antiparticle (say positron) have been well established as C $\mathrm{P}$ (charged parity) violation. Asseo and Sol 44 speculated the large-scale inter galactic magnetic field and is of primordial origin at present measure $10^{-8} \mathrm{G}$ and gives rise to a density of order $10^{-35} \mathrm{gcm}^{-3}$. The present day magnitude of magnetic energy is very small in comparison with the estimated matter density, it might not have been negligible during early stage of evolution of the universe. FRW models are approximately valid as present day magnetic field is very small. The existence of a primordial magnetic field is limited to Bianchi Types I, II, III, $V I_{0}$ and $V I I_{0}$ as shown by Hughston and Jacobs [45]. Large-scale magnetic fields give rise to anisotropies in the universe. The anisotropic pressure created by the magnetic fields dominates the evolution of the shear anisotropy and it decays slower than if the pressure was isotropic [46, 47]. Such fields can be 
generated at the end of an inflationary epoch [48]-50]. Anisotropic magnetic field models have significant contribution in the evolution of galaxies and stellar objects.

\section{The metric and field equations}

We consider the metric in the form of Marder [51]

$$
d s^{2}=A^{2}\left(d x^{2}-d t^{2}\right)+B^{2} d y^{2}+C^{2} d z^{2},
$$

where the metric potential $A, B$ and $C$ are functions of $x$ and $t$. The energy momentum tensor is taken as

$$
T_{i}^{j}=(\rho+p) v_{i} v^{j}+p g_{i}^{j}+E_{i}^{j},
$$

where $E_{i}^{j}$ is the electro-magnetic field given by Lichnerowicz [52] as

$$
E_{i}^{j}=\bar{\mu}\left[h_{l} h^{l}\left(v_{i} v^{j}+\frac{1}{2} g_{i}^{j}\right)-h_{i} h^{j}\right] .
$$

Here $\rho$ and $p$ are the energy density and isotropic pressure respectively and $v^{i}$ is the flow vector satisfying the relation

$$
g_{i j} v^{i} v^{j}=-1
$$

$\bar{\mu}$ is the magnetic permeability and $h_{i}$ the magnetic flux vector defined by

$$
h_{i}=\frac{1}{\bar{\mu}}{ }^{*} F_{j i} v^{j},
$$

where ${ }^{*} F_{i j}$ is the dual electro-magnetic field tensor defined by Synge 53 ]

$$
{ }^{*} F_{i j}=\frac{\sqrt{-g}}{2} \epsilon_{i j k l} F^{k l} .
$$

$F_{i j}$ is the electro-magnetic field tensor and $\epsilon_{i j k l}$ is the Levi-Civita tensor density. The coordinates are considered to be comoving so that $v^{1}=0=v^{2}=v^{3}$ and $v^{4}=\frac{1}{A}$. We consider that the current is flowing along the z-axis so that $h_{3} \neq 0$, $h_{1}=0=h_{2}=h_{4}$. The only non-vanishing component of $F_{i j}$ is $F_{12}$. The Maxwell's equations

$$
F_{i j ; k}+F_{j k ; i}+F_{k i ; j}=0,
$$

and

$$
\left[\frac{1}{\bar{\mu}} F^{i j}\right]_{; j}=0,
$$

require that $F_{12}$ be function of $x$ alone. We assume that the magnetic permeability as a function of $x$ and $t$ both. Here the semicolon represents a covariant 
differentiation.

The Einstein's field equations ( in gravitational units $\mathrm{c}=1, \mathrm{G}=1$ ) read as

$$
R_{i}^{j}-\frac{1}{2} R g_{i}^{j}+\Lambda g_{i}^{j}=-8 \pi T_{i}^{j},
$$

for the line element (1) has been set up as

$$
\begin{gathered}
8 \pi A^{2}\left(p+\frac{F_{12}^{2}}{2 \bar{\mu} A^{2} B^{2}}\right)=-\frac{B_{44}}{B}-\frac{C_{44}}{C}+\frac{A_{4}}{A}\left(\frac{B_{4}}{B}+\frac{C_{4}}{C}\right) \\
+\frac{A_{1}}{A}\left(\frac{B_{1}}{B}+\frac{C_{1}}{C}\right)+\frac{B_{1} C_{1}}{B C}-\frac{B_{4} C_{4}}{B C}-\Lambda A^{2}, \\
8 \pi A^{2}\left(p+\frac{F_{12}^{2}}{2 \bar{\mu} A^{2} B^{2}}\right)=-\left(\frac{A_{4}}{A}\right)_{4}+\left(\frac{A_{1}}{A}\right)_{1}-\frac{C_{44}}{C}+\frac{C_{11}}{C}-\Lambda A^{2}, \\
8 \pi A^{2}\left(p-\frac{F_{12}^{2}}{2 \bar{\mu} A^{2} B^{2}}\right)=-\left(\frac{A_{4}}{A}\right)_{4}+\left(\frac{A_{1}}{A}\right)_{1}-\frac{B_{44}}{B}+\frac{B_{11}}{B}-\Lambda A^{2}, \\
8 \pi A^{2}\left(\rho+\frac{F_{12}^{2}}{2 \bar{\mu} A^{2} B^{2}}\right)=-\frac{B_{11}}{B}-\frac{C_{11}}{C}+\frac{A_{1}}{A}\left(\frac{B_{1}}{B}+\frac{C_{1}}{C}\right) \\
+\frac{A_{4}}{A}\left(\frac{B_{4}}{B}+\frac{C_{4}}{C}\right)-\frac{B_{1} C_{1}}{B C}+\frac{B_{4} C_{4}}{B C}+\Lambda A^{2}, \\
0=\frac{B_{14}}{B}+\frac{C_{14}}{C}-\frac{A_{1}}{A}\left(\frac{B_{4}}{B}+\frac{C_{4}}{C}\right)-\frac{A_{4}}{A}\left(\frac{B_{1}}{B}+\frac{C_{1}}{C}\right),
\end{gathered}
$$

where the sub indices 1 and 4 in $\mathrm{A}, \mathrm{B}, \mathrm{C}$ and elsewhere indicate ordinary differentiation with respect to $x$ and $t$, respectively.

\section{Solution of the field equations}

Equations (10) - (12) lead to

$$
\begin{gathered}
\left(\frac{A_{4}}{A}\right)_{4}-\frac{B_{44}}{B}+\frac{A_{4}}{A}\left(\frac{B_{4}}{B}+\frac{C_{4}}{C}\right)-\frac{B_{4} C_{4}}{B C}= \\
\left(\frac{A_{1}}{A}\right)_{1}+\frac{C_{11}}{C}-\frac{A_{1}}{A}\left(\frac{B_{1}}{B}+\frac{C_{1}}{C}\right)-\frac{B_{1} C_{1}}{B C}=\mathrm{a} \text { (constant), }
\end{gathered}
$$

and

$$
\frac{8 \pi F_{12}^{2}}{\bar{\mu} B^{2}}=\frac{B_{44}}{B}-\frac{B_{11}}{B}+\frac{C_{11}}{C}-\frac{C_{44}}{C} .
$$

Eqs. (10) - (14) represent a system of five equations in seven unknowns $A$, $B, C, \rho, p, \Lambda$ and $\bar{\mu}$. For the complete determination of these unknowns two more conditions are needed. As in the case of general-relativistic cosmologies, 
the introduction of inhomogeneities into the cosmological equations produces a considerable increase in mathematical difficulty: non-linear partial differential equations must now be solved. In practice, this means that we must proceed either by means of approximations which render the non-linearities tractable, or we must introduce particular symmetries into the metric of the space-time in order to reduce the number of degrees of freedom which the inhomogeneities can exploit. In the present case, we assume that the metric is Petrov type-II non-degenerate. This requires that

$$
\begin{gathered}
\left(\frac{B_{11}+B_{44}+2 B_{14}}{B}\right)-\left(\frac{C_{11}+C_{4}+2 C_{14}}{C}\right)= \\
\frac{2\left(A_{1}+A_{4}\right)\left(B_{1}+B_{4}\right)}{A B}-\frac{2\left(A_{1}+A_{4}\right)\left(C_{1}+C_{4}\right)}{A C} .
\end{gathered}
$$

Let us consider that

$$
\begin{aligned}
& A=f(x) \lambda(t), \\
& B=g(x) \mu(t), \\
& C=g(x) \nu(t) .
\end{aligned}
$$

Using (18) in (14) and (17), we get

$$
\left[\frac{\frac{g_{4}}{g}-\frac{f_{1}}{f}}{\frac{g_{1}}{g}}\right]=\left[\frac{\frac{2 \lambda_{4}}{\lambda}}{\frac{\mu_{4}}{\mu}+\frac{\nu_{4}}{\nu}}\right]=\mathrm{b} \text { (constant), }
$$

and

$$
\frac{\frac{\mu_{44}}{\mu}-\frac{\nu_{44}}{\nu}}{\frac{\mu_{4}}{\mu}-\frac{\nu_{4}}{\nu}}-\frac{2 \lambda_{4}}{\lambda}=2\left(\frac{f_{1}}{f}-\frac{g_{1}}{g}\right)=\mathrm{L} \text { (constant). }
$$

Equation (19) leads to

$$
f=n g^{(1-b)}
$$

and

$$
\lambda=m(\mu \nu)^{\frac{b}{2}},
$$

where $m$ and $n$ are constants of integration. Equations (15), (18) and (20) lead to

$$
\left(\frac{b}{2}-1\right) \frac{\mu_{44}}{\mu}+(b-1) \frac{\mu_{4} \nu_{4}}{\mu \nu}=a
$$

and

$$
(2-b) \frac{g_{11}}{g}+(3 b-4) \frac{g_{1}^{2}}{g^{2}}=a .
$$

Let us assume

$$
\mu=e^{U+W},
$$

and

$$
\nu=e^{U-W} .
$$


Equations (20), (25) and (26) lead to

$$
W_{4}=M e^{L t+2(b-1) U},
$$

where $\mathrm{M}$ is constant. From equations (23), (25), (26) and (27), we have

$$
(b-1) U_{44}+2(b-1) U_{4}^{2}-2 b M e^{L t+2(b-1) U} U_{4}-M L e^{L t+2(b-1) U}=a .
$$

If we put $e^{2 U}=\xi$ in equation (28), we obtain

$$
\frac{(b-1)}{2} \frac{d^{2} \xi}{d t^{2}}-M \frac{d}{d t}\left(e^{L t} \xi^{b}\right)=a \xi .
$$

If we consider $\xi=e^{h t}$, then equation (29) leads to

$$
\frac{(b-1)}{2} g^{2} e^{h t}-M \frac{d}{d t}\left(e^{L t} e^{h b t}\right)=a e^{h t},
$$

which again reduces to

$$
h=\frac{L}{1-b}
$$

and

$$
a=\frac{L(L+2 M)}{2(b-1)}
$$

Thus

$$
U=\frac{L t}{2(1-b)} .
$$

Equations (27) and (33) reduce to

$$
W=M t+\log N,
$$

where $N$ is an integrating constant. Eq. (24) leads to

$$
g=\beta \sinh ^{\frac{2-b}{2(b-1)}}(\alpha x+\delta),
$$

where

$$
\alpha=\frac{\sqrt{2 a(b-1)}}{(2-b)}, \quad \beta=\beta_{0}^{\frac{2-b}{2(b-1)}}
$$

and $\beta_{0}, \delta$ being constants of integration. Hence

$$
\begin{gathered}
f=n \beta \sinh ^{\frac{b-2}{2(b-1)}}(\alpha x+\delta), \\
\lambda=m e^{\frac{L b t}{2(1-b)}}, \\
\mu=e^{\frac{L b t}{2(1-b)}+M t+\log N}, \\
\nu=e^{\frac{L b t}{2(1-b)}-M t-\log N} .
\end{gathered}
$$


Therefore, we have

$$
\begin{gathered}
A=f \lambda=m n \beta e^{\frac{L b t}{2(1-b)}} \sinh ^{\frac{b-2}{2}}(\alpha x+\delta), \\
B=g \mu=N \beta e^{\left(\frac{L}{1-b}+2 M\right) \frac{t}{2}} \sinh ^{\frac{2-b}{2(b-1)}}(\alpha x+\delta), \\
C=g \nu=\frac{\beta}{N} e^{\left(\frac{L}{1-b}-2 M\right) \frac{t}{2}} \sinh ^{\frac{2-b}{2(b-1)}}(\alpha x+\delta) .
\end{gathered}
$$

By using the transformation

$$
\begin{gathered}
\alpha X=\alpha x+\delta, \\
Y=G y, \\
Z=H z, \\
T=t,
\end{gathered}
$$

the metric (11) reduces to the form

$$
\begin{gathered}
d s^{2}=K^{2} \sinh ^{b-2}(\alpha X) e^{\frac{L T b}{1-b}}\left(d X^{2}-d T^{2}\right)+ \\
\sinh ^{\frac{2-b}{b-1}}(\alpha X) e^{\left(\frac{L}{1-b}+2 M\right) T} d Y^{2}+\sinh ^{\frac{2-b}{b-1}}(\alpha X) e^{\left(\frac{L}{1-b}-2 M\right) T} d Z^{2},
\end{gathered}
$$

where $K=m n \beta, G=N \beta$ and $H=\frac{\beta}{N}$.

\section{Some Physical and Geometric Features}

The physical parameters, pressure $(p)$ and density $(\rho)$, for the model (44) are given by

$$
\begin{gathered}
8 \pi p=\frac{1}{K^{2}} e^{\frac{L b T}{b-1}} \sinh ^{2-b}(\alpha X)\left[\frac{(2-b)^{2} \alpha^{2}}{4(b-1)}\left\{1+\frac{2-b}{b-1} \operatorname{coth}^{2}(\alpha X)\right\}\right. \\
\left.-\frac{L^{2}}{4(1-b)^{2}}-M^{2}\right]-\Lambda \\
8 \pi \rho=\frac{1}{K^{2}} e^{\frac{L b T}{b-1}} \sinh ^{2-b}(\alpha X)\left[\frac{(2-b) \alpha^{2}}{2(b-1)}\left\{\frac{b}{b-1} \operatorname{coth}^{2}(\alpha X)-1\right\}\right. \\
\left.+\frac{L^{2}(2 b-1)}{4(1-b)^{2}}-M^{2}-\frac{M L}{(1-b)}\right]+\Lambda .
\end{gathered}
$$

In this case to find the explicit value of cosmological constant $\Lambda(t)$, one may assume that the fluid obey an equation of state of the form

$$
p=\gamma \rho
$$




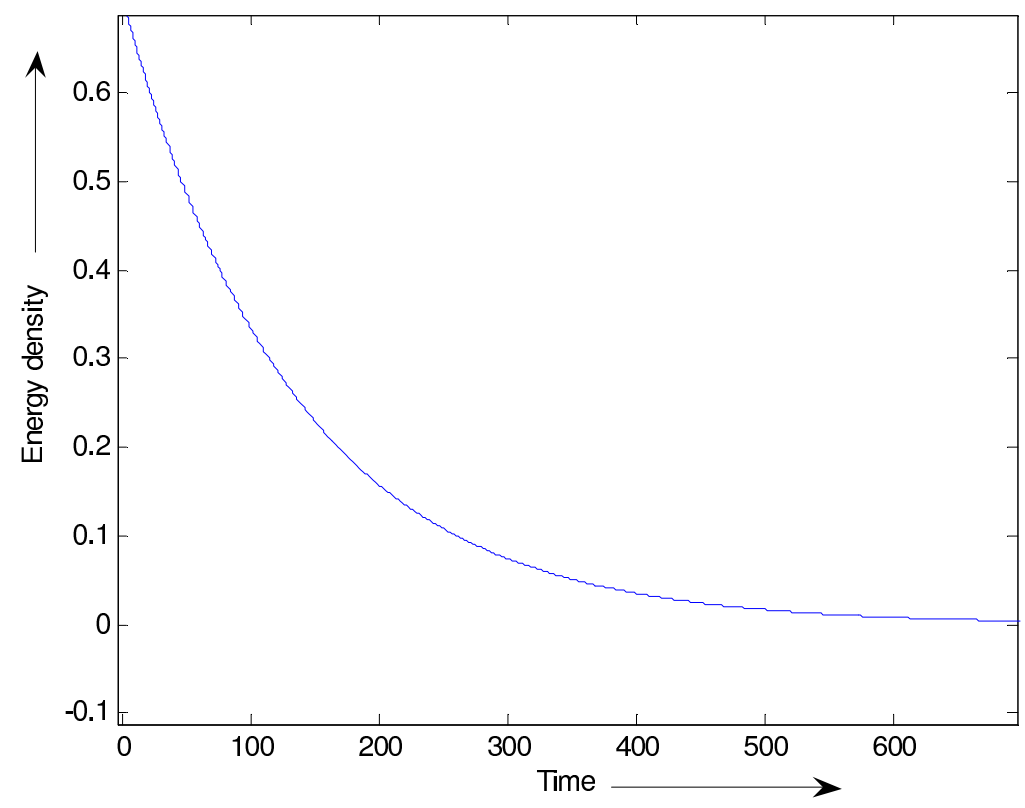

Figure 1: The plot of energy density $\rho$ vs. time T with parameters $b=0.5, m=$ $0.03, n=0.25, K=0.2$ and $\gamma=0.4$

where $\gamma(0 \leq \gamma \leq 1)$ is a constant.

Using equation (47) in (45) and then solving with(46), we have

$$
\begin{aligned}
8 \pi(1+\gamma) \rho= & \frac{1}{K^{2}} e^{\frac{L b T}{b-1}} \sinh ^{2-b}(\alpha X)\left[\frac{(2-b)\left(b^{2}-2 b+4\right) \alpha^{2}}{4(b-1)^{2}} \operatorname{coth}^{2}(\alpha X)\right. \\
& \left.+\frac{b(b-2) \alpha^{2}}{4(b-1)}-\frac{L^{2}}{2(1-b)}-2 M^{2}-\frac{M L}{(1-b)}\right]
\end{aligned}
$$

Eliminating $\gamma$ from (46) and (48), we obtain

$$
\begin{gathered}
(1+\gamma) \Lambda=\frac{1}{K^{2}} e^{\frac{L b T}{b-1}} \sinh ^{2-b}(\alpha X)\left[\frac{(2-b)\left(b^{2}-4 \gamma b+4(b+1)\right) \alpha^{2}}{4(b-1)^{2}} \operatorname{coth}^{2}(\alpha X)\right. \\
\left.-\frac{(b-2)(2+2 \gamma-b) \alpha^{2}}{4(b-1)}-\frac{L^{2}(\gamma(2 b-1)+1)}{4(1-b)^{2}}-(1-\gamma) M^{2}+\frac{\gamma M L}{(1-b)}\right],
\end{gathered}
$$

From (46) and (48), we note that $\rho(t)$ is the decreasing function of time and $\rho>0$ for all time. This behaviour is clearly shown in figure 1 , as a representative case with appropriate choice of constants of integration and other physical parameters using reasonably well known situations. 


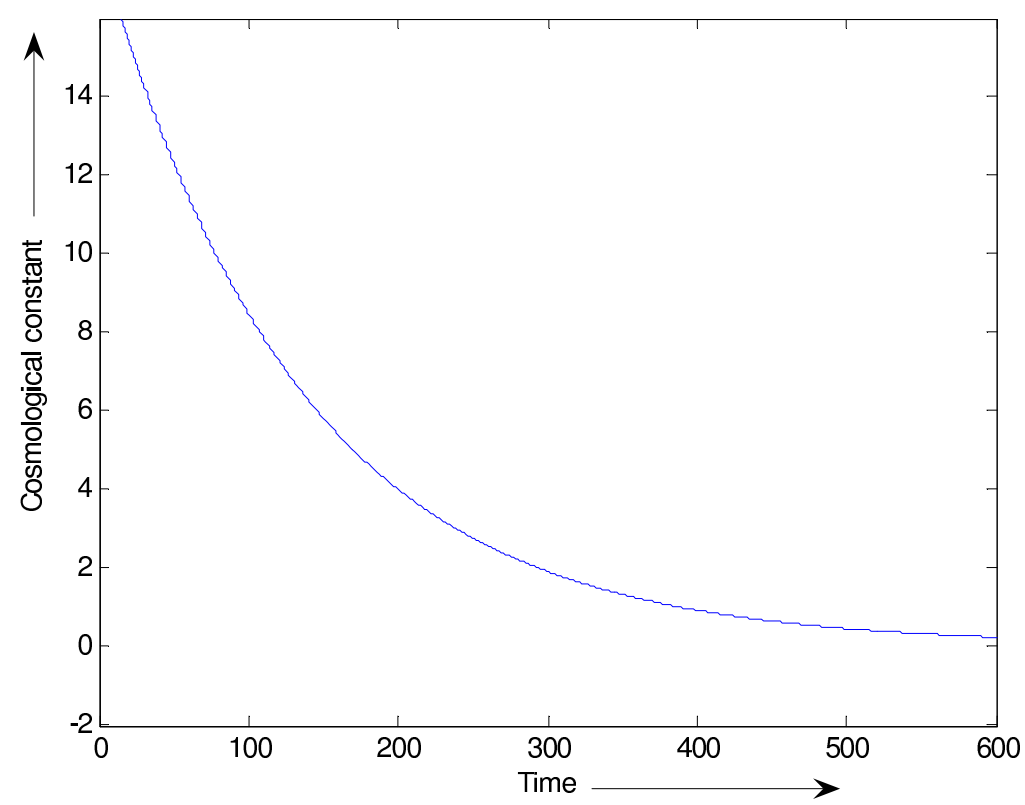

Figure 2: The plot of cosmological constant $\Lambda$ vs. time $\mathrm{T}$ with parameters $b=0.5, m=0.03, n=0.25, K=0.2$ and $\gamma=0.4$

In spite of homogeniety at large scale our universe is inhomogenrous at small scale, so physical quantities being position dependent are more natural in our observable universe if we do not go to super high scale. This result show this kind of physical importance. In recent time theoreticians and observers draw high attention on $\Lambda$ - terms due to various reasons. The non-trivial role of the vacuum in early universe generates a $\Lambda$ that leads to inflationary phase. Obsevationally this term provides an additional parameter to accommodate conflicting data on the value of Hubble's constant, deceleration parameter, the density parameters and the age of universe [54 and [55. Assuming that $\Lambda$ owes it origin to vacuum interactions, as suggested in particular by Sakharav [56], it follows that it would in general be a function of space and time co-ordinates, rather than a strict constant. In a homogeneous universe $\Lambda$ will be most time dependent [57. In our case this approach can generate $\Lambda$ that varies both with space and time. In considering the nature of local massive objects, however the space dependence of $\Lambda$ can not ignored. For detail discussion, the readers are advised to see the reference (Narlikar, Pecker and Vigier [58, Ray and Ray [59], Tiwari, Ray and Bhadra 60]).

From (49), we see that cosmological constant $\Lambda$ is a decreasing function of time and it approaches a small positive value at late time. This behaviour is clearly shown in figure 2 . Recent cosmological obsevations suggest the existence of positive cosmological constant $\Lambda$ with the magnitude $\Lambda\left(\frac{G \hbar}{c^{3}}\right) \approx 10^{-123}$. These 
observations on magnitude and red-shift of type Ia supernova suggest that our universe may be an accelerating one with induced cosmological density through the cosmological $\Lambda$-term. Thus the model presented in this paper is consistent with the results of recent observations.

The non-vanishing component $F_{12}$ of the electromagnetic field tensor is given by

$$
F_{12}=\sqrt{\frac{\bar{\mu}}{8 \pi} \frac{2 M L}{(1-b)}} G e^{\left(\frac{L}{1-b}+2 M\right) \frac{T}{2}} \sinh ^{\frac{2-b}{2(b-1)}}(\alpha X),
$$

where $\bar{\mu}$ remains undetermined as function of $x$ and $t$ both.

The scalar of expansion $(\theta)$ calculated for the flow vector $\left(v^{i}\right)$ is given by

$$
\theta=\frac{L(b+2)}{2 K(1-b)} e^{\frac{L b T}{2(b-1)}} \sinh ^{\frac{(2-b)}{2}}(\alpha X) .
$$

The shear scalar $\left(\sigma^{2}\right)$, acceleration vector $\left(\dot{v}_{i}\right)$, deceleration parameter $q$, proper volume $V$ and Hubble parameter $H$ for the model (45) are given by

$$
\begin{gathered}
\sigma^{2}=\frac{\left(L^{2}+12 M^{2}\right)}{12 K^{2}} e^{\frac{L b T}{(b-1)}} \sinh ^{(2-b)}(\alpha X), \\
\dot{v}_{i}=\left(\frac{1}{2}(b-2) \alpha \operatorname{coth}(\alpha X), 0,0,0\right), \\
q=-\frac{4 K^{2}(b+1)^{2}}{81(b+2)^{2}} \exp \left(\frac{L b T}{1-b}\right) \sinh ^{(b-2)}(\alpha X), \\
V=\sqrt{-g}=K^{2} G H e^{\frac{L(b+1) T}{(1-b)}} \sinh ^{\frac{(b-2)(b-3)}{(b-1)}}(\alpha X), \\
H=\frac{3 L(b+2)}{2 K(1-b)} e^{\frac{L b T}{2(b-1)}} \sinh ^{\frac{(2-b)}{2}}(\alpha X) .
\end{gathered}
$$

From equations(51) and (52), we have

$$
\frac{\sigma^{2}}{\theta^{2}}=\frac{\left(L^{2}+12 M^{2}\right)\left(1-b^{2}\right)}{3 L^{2}(b+2)^{2}}=\text { constant. }
$$

The rotation $\omega$ is identically zero and the non-vanishing component of conformal curvature tensor are given by

$$
\begin{gathered}
C_{(1212)}=\frac{1}{6 K^{2}} e^{\frac{L b T}{(b-1)}} \sinh ^{(2-b)}(\alpha X)\left[b \alpha-\frac{L^{2}}{4 b}+3 M L-2 M^{2}\right], \\
C_{(1313)}=\frac{1}{6 K^{2}} e^{\frac{L b T}{(b-1)}} \sinh ^{(2-b)}(\alpha X)\left[b \alpha-\frac{L^{2}}{4 b}-3 M L-2 M^{2}\right], \\
C_{(2323)}=\frac{1}{3 K^{2}} e^{\frac{L b T}{(b-1)}} \sinh ^{(2-b)}(\alpha X)\left[-b \alpha+\frac{L^{2}}{4 b}+\frac{M L}{(1-b)}+2 M^{2}\right], \\
C_{(1224)}=\frac{M L}{2 K^{2}} e^{\frac{L b T}{(b-1)}} \sinh ^{(2-b)}(\alpha X) .
\end{gathered}
$$


The reality conditions (Ellis [61])

$$
\text { (i) } \rho+p>0, \quad \text { (ii) } \rho+3 p>0,
$$

lead to

$$
\begin{gathered}
\frac{(2-b) \alpha^{2}}{4(b-1)^{2}}\left[\left(b^{2}-2 b+4\right) \operatorname{coth}^{2}(\alpha X)+b(1-b)\right]> \\
\frac{L^{2}}{2(1-b)}+2 M^{2}+\frac{M L}{(1-b)},
\end{gathered}
$$

and

$$
\begin{gathered}
e^{\frac{L b T}{(b-1)}}\left[\frac{(2-b)\left(3 b^{2}-10 b+12\right) \alpha^{2}}{4(b-1)^{2}} \operatorname{coth}^{2}(\alpha X)+\frac{(2-b)(3 b-4) \alpha^{2}}{4(1-b)}\right. \\
\left.+\frac{L^{2}(2 b-4)}{4(1-b)^{2}}-\frac{M L}{(1-b)}-4 M^{2}\right]+2 K^{2} \Lambda \sinh ^{(b-2)}(\alpha X)>0
\end{gathered}
$$

respectively.

The dominant energy condition is given by Hawking and Ellis 62 .

$$
\text { (i) } \rho-p \geq 0, \quad \text { (ii) } \quad \rho+p \geq 0
$$

lead to

$$
\begin{gathered}
e^{\frac{L b T}{(b-1)}}\left[\frac{(2-b) \alpha^{2}}{2(b-1)}\left\{\frac{\left(b^{2}-6 b+4\right)}{2(1-b)} \operatorname{coth}^{2}(\alpha X)+\frac{b-4}{2}\right\}\right. \\
\left.+\frac{L^{2} b}{2(1-b)^{2}}-\frac{M L}{(1-b)}\right]+2 K^{2} \Lambda \sinh ^{(b-2)}(\alpha X) \geq 0
\end{gathered}
$$

and

$$
\begin{gathered}
\frac{(2-b) \alpha^{2}}{4(b-1)^{2}}\left[\left(b^{2}-2 b+4\right) \operatorname{coth}^{2}(\alpha X)+b(1-b)\right] \geq \\
\frac{L^{2}}{2(1-b)}+2 M^{2}+\frac{M L}{(1-b)},
\end{gathered}
$$

respectively.

\section{Thermodynamical behaviour and entropy of universe}

From the thermodynamics [63, 64, we apply the combination of first and second law of thermodynamics to the system with volume V. As we know that

$$
\mathrm{T} d S=d(\rho V)+p d V
$$


where T, S represents the tempreture and entropy respectively.

Eq. (66) may be written as

$$
\underset{3}{\mathrm{~T}} d S=d[(\rho+p) V]-V d p
$$

The integrability condition is necessary to define a perefect fluid as a thermodynamical syetem [65, 66. It is given by

$$
d p=\frac{\rho+P}{\mathrm{~T}} d \mathrm{~T}
$$

Plugging eq. (68) in eq. (67), we have the differential equation

$$
d S=\frac{1}{\mathrm{~T}} d[(\rho+p) V]-(\rho+p) V \frac{d \mathrm{~T}}{\mathrm{~T}^{2}}
$$

we rewrite eq (69) as

$$
d S=d\left[\frac{(\rho+p) V}{\mathrm{~T}}+c\right]
$$

where $\mathrm{c}$ is constant.

Hence the entropy is defined as

$$
S=\frac{\rho+p}{\underset{3}{\mathrm{~T}}} V
$$

Let the entropy density be s, so that

$$
s=\frac{S}{V}=\frac{\rho+p}{\mathrm{~T}}=\frac{(1+\gamma) \rho}{\mathrm{T}}
$$

where $p=\gamma \rho$ and $0<\gamma \leq 1$.

If we define the entropy density in terms of temprature then the first law of thermodynamics may be written as

$$
d(\rho V)+\gamma \rho d V=(1+\rho) \mathrm{T} d\left(\frac{\rho V}{\mathrm{~T}}\right)
$$

which on integration yields

$$
\mathrm{T}=c_{0} \rho^{\frac{\gamma}{(1+\gamma)}}
$$

where $c_{0}$ is constant of integration.

From eqs. (72) and (71), we obtain

$$
s=\left(\frac{1+\gamma}{c_{0}}\right) \rho^{\frac{1}{1+\rho}}
$$

These equation are not valid for $\gamma=-1$. For the Zel'dovich fluid $(\gamma=1)$, we get

$$
\mathrm{T}=c_{0} \rho^{\frac{1}{2}}
$$




$$
\begin{gathered}
s=\frac{2}{c_{0}} \rho^{\frac{1}{2}} \\
\Rightarrow s \sim \rho^{\frac{1}{2}} \sim \mathrm{T}
\end{gathered}
$$

Thus the entropy density is proportional to the tempreture.

Now the tempreture, entropy density and entropy of Zel'dovich universe is given by

$$
\begin{gathered}
\text { T }=T_{0}\left[\frac { 1 } { K ^ { 2 } } e ^ { \frac { L b T } { b - 1 } } \operatorname { s i n h } ^ { 2 - b } ( \alpha X ) \left[\frac{(2-b) \alpha^{2}}{2(b-1)}\left\{\frac{b}{b-1} \operatorname{coth}^{2}(\alpha X)-1\right\}\right.\right. \\
\left.\left.+\frac{L^{2}(2 b-1)}{4(1-b)^{2}}-M^{2}-\frac{M L}{(1-b)}\right]+\Lambda\right]^{\frac{1}{2}} . \\
s=s_{0}\left[\frac { 1 } { K ^ { 2 } } e ^ { \frac { L b T } { b - 1 } } \operatorname { s i n h } ^ { 2 - b } ( \alpha X ) \left[\frac{(2-b) \alpha^{2}}{2(b-1)}\left\{\frac{b}{b-1} \operatorname{coth}^{2}(\alpha X)-1\right\}\right.\right. \\
\left.\left.+\frac{L^{2}(2 b-1)}{4(1-b)^{2}}-M^{2}-\frac{M L}{(1-b)}\right]+\Lambda\right]^{\frac{1}{2}} \cdot \\
\left.\left.\quad+\frac{L^{2}(2 b-1)}{4(1-b)^{2}}-M^{2}-\frac{M L}{(1-b)}\right]+\Lambda\right]^{\frac{1}{2}} .
\end{gathered}
$$

where $T_{0}=\frac{c_{0}}{\sqrt{8 \pi}}, s_{0}=\frac{2}{c_{0} \sqrt{8 \pi}}$ and $S_{0}=s_{0} K$ are constant.

For radiating fluid $\left(\gamma=\frac{1}{3}\right)$, we get

$$
\begin{gathered}
\mathrm{T} \sim \rho^{\frac{1}{4}} \\
s \sim \rho^{\frac{3}{4}} \sim \mathrm{T}^{3}
\end{gathered}
$$

Thus the entropy density is proportional to cube of tempreture.

Now the tempreture, entropy density and entropy of radiating universe is given by

$$
\begin{gathered}
\text { T }=T_{00}\left[\frac { 1 } { K ^ { 2 } } e ^ { \frac { L b T } { b - 1 } } \operatorname { s i n h } ^ { 2 - b } ( \alpha X ) \left[\frac{(2-b) \alpha^{2}}{2(b-1)}\left\{\frac{b}{b-1} \operatorname{coth}^{2}(\alpha X)-1\right\}\right.\right. \\
\left.\left.+\frac{L^{2}(2 b-1)}{4(1-b)^{2}}-M^{2}-\frac{M L}{(1-b)}\right]+\Lambda\right]^{\frac{1}{4}} \cdot \\
s=s_{00}\left[\frac { 1 } { K ^ { 2 } } e ^ { \frac { L b T } { b - 1 } } \operatorname { s i n h } ^ { 2 - b } ( \alpha X ) \left[\frac{(2-b) \alpha^{2}}{2(b-1)}\left\{\frac{b}{b-1} \operatorname{coth}^{2}(\alpha X)-1\right\}\right.\right.
\end{gathered}
$$




$$
\begin{gathered}
\left.\left.+\frac{L^{2}(2 b-1)}{4(1-b)^{2}}-M^{2}-\frac{M L}{(1-b)}\right]+\Lambda\right]^{\frac{3}{4}} . \\
S=S_{00} e^{\frac{L(b+1) T}{(1-b)}} \sinh ^{\frac{(b-2)(b-3)}{(b-1)}}(\alpha X)\left[\frac{1}{K^{2}} e^{\frac{L b T}{b-1}} \sinh ^{2-b}(\alpha X)\right. \\
\left.\left[\frac{(2-b) \alpha^{2}}{2(b-1)}\left\{\frac{b}{b-1} \operatorname{coth}^{2}(\alpha X)-1\right\}+\frac{L^{2}(2 b-1)}{4(1-b)^{2}}-M^{2}-\frac{M L}{(1-b)}\right]+\Lambda\right]^{\frac{3}{4}} .
\end{gathered}
$$

where $T_{00}=\frac{c_{0}}{(8 \pi)^{1 / 2}}, s_{00}=\frac{2}{c_{0}(8 \pi)^{1 / 2}}$ and $S_{00}=s_{0} K^{2}$ are constant.

\section{Discussion and Concluding Remarks}

The present study deals the plane-symmetric inhomogeneous cosmological model of electro-magnetic perfect fluid as the source of matter. FRW models are appoximatly valid, as present day magnetic field is very small. Maarteens [67] in his study explained that magnetic fields are observed not only in stars but also in galaxies. In princple, these fields could play a significant role in structure formation but also affect the anisotropies in cosmic microwave background radiation $[\mathrm{CMB}]$. Since the electric and magnetic fields are interrelated, their independent nature disappears when we consider them as time dependance. Hence, it would be proper to look upon these fields as a single field - electromagnetic field. Generally the model represents expanding, shearing, non-rotating and Petrov type-II non-degenerate universe in which the flow vector is geodesic. We find that the model starts expanding at $T=0$ and goes on expanding indefinitely. For large values of $T$, the model is conformally flat and Petrov type-II nondegenerate. Since $\frac{\sigma}{\theta}=$ constant, hence the model does not approach isotropy. The value of cosmological constant is found to be small and pasitive supported by recent results from the supernovae observations recently obtained by High-Z Supernovae Ia Team and Supernovae Cosmological Project. The relation between cosmological constant and thermodynamical quantities is established and from eqs. (82) and (85), it is clear that cosmological constant affect entropy. The electromagnetic field tensor does not vanish when $L \neq 0, M \neq 0$, and $b \neq 1$. For large values of $T$ and $L+2 M(1-b)<0, F_{12}$ tends to zero. For $b=1$, we obtain singularity and model approach isotropy. $b<1$ and $b>1$ imposed the restriction on the value of $M$ and $L$ which affect all the physical and kinematical parameters of the model.

In spite of homogeneity at large scale our universe is inhomogeneous at small scale, so physical quantities being position-dependent are more natural in our observable universe if we do not go to super high scale. Our derived model shows this kind of physical importance. The expressions for deceleration parameter $(q)$ and Hubble parameter $(H)$ given by Eqs. (54) and (56) respectively are functions of $\mathrm{x}$ and $\mathrm{t}$ as in the case of inhomogeneous cosmological models. Obviously 
these two expressions are different from that of FRW cosmological model. The present study also extend the work of Yadav and Bagora 68 for inhomogeneous universe with varying $\Lambda$ and clarify thermodynamics of inhomogeneous universe by introducing the integrability condition and tempreture. It is found that $s \sim \rho^{\frac{1}{2}} \sim \mathrm{T}$ and $s \sim \rho^{\frac{3}{4}} \sim \mathrm{T}_{3}^{3}$, for Zeldo'vich and radiating fluid model respectively. From these expressions it is clear that $\rho$ is the function of tempreture and volume, thus a new general equation of state describing the Zel'dovich fluid and radiating fluid models as a function of tempreture and volume is found. The basic equations of thermodynamics for inhomogeneous universe has been deduced which may be useful for better understanding of evolution of universe.

\section{References}

[1] G. Efstathiou, W.J.Sutherland and S.J. Maddox, Nature(London) 348 (1990) 705.

[2] L.A. Kofman, N.Y.Gnedin and N. A. Bahcall,Astrophys. J. 413 (1993) 1.

[3] M. Ozer and M. O. Taha Phys. Lett. B 171 (1987) 363.

[4] K. Freese,F. C. Adams, J. A. Frieman and E Mottola Nucl. Phys. B 287 (1987) 776 .

[5] M. Gasperini, Phys. Lett. B 194 (1987) 347.

[6] W. Chen and Y. S. Wu, Phys. Rev. D 41 (1990) 695.

[7] A-M M. Abdel-Rahman, Phys. Rev. D 45 (1992) 3497.

[8] J. C. Carvalho ,J. A. S. Lima and I. Waga, Phys. Rev.D 46 (1992) 2404.

[9] J. M. Salim and I. Waga Class. Quant. Grav. 10 (1993) 1767.

[10] M. S. Berman, Phys. Rev. D 43 (1991) 1075.

[11] T. S. Olson and T. F. Jordan, Phys. Rev. D 35 (1987) 3258.

J. Matyjasek Phys. Rev. D 51 (1995) 4154.

[12] Hu B L and Parkar L 1977 Phys. Lett. 63A 217.

[13] Hu B L, Phys. Lett. 97A (1983) 368.

Nesteruk A V and Ottewill A C, Class.quantum Grav. 12 (1995) 51.

[14] B. Ratra and P. J. E. Peebles, Phys. Rev. D 37 (1988) 3406.

[15] V. Sahni and A. Starobinsky, Int. J. Mod. Phys. D 9 (2000) 373.

[16] S. M. Carroll, W. H. Press and E. L. Turner, Ann. Rev. Astron. Astrophys. 30 (1992) 499.

[17] P. J. E. Peebles, Rev. Mod. Phys. 75 (2003) 559. 
[18] T. Padmanabhan, Phys. Rep. 380 (2003) 235.

T. Padmanabhan, Dark Energy and Gravity, gr-qc/0705.2533 (2007).

[19] S. Perlmutter et al., Astrophys. J. 483 (1997) 565;

S. Perlmutter et al., Nature 391 (1998) 51;

S. Perlmutter et al., Astrophys. J. 517 (1999) 565.

[20] A. G. Riess et al., Astron. J. 116 (1998) 1009;

A. G. Riess et al., Astrophys. J. 560 (2001) 49;

A. G. Riess et al., Astrophys. J. 607 (2004) 665;

A. G. Riess et al., Astrophys. J. 659 (2007) 98.

[21] P. M. Garnavich et al., Astrophys. J. 493 (1998) L53;

P. M. Garnavich et al., Astrophys. J. 509 (1998) 74.

[22] B. P. Schmidt et al., Astrophys. J. 507 (1998) 46.

[23] J. P. Blakeslee et al., Astrophys. J. 589 (2003) 693.

[24] P. Astier et al., Astron. Astrophys. 447 (2006) 31.

[25] D. N. Spergel et al., Astrophys. J. Suppl. 170 (2007) 377.

[26] D. J. Eisentein et al., Astrophys. J. 633 (2005) 560.

[27] S. M. Carroll, eConf C0307282, TTH09 (2003) [AIP Conf. Proc. 743 (2005) 16]

[28] E. W. Kolb, S. Matarrese and A. Riotto, New J. Phys. 8 (2006) 322;

E. W. Kolb, S. Matarrese, A. Notari and A. Riotto, arXiv:hep-th/0503117

[29] A. Upadhye, M. Ishak and P. J. Steinhardt, Phys. Rev. D 72 (2005) 063501.

[30] R.C. Tolman, Proc. Nat. Acad. Sci. (USA) 20 (1934) 169.

[31] H. Bondi, Mon. Not. R. Astro. Soc. 107 (1947) 410.

[32] A.H. Taub, Ann. Math. 53 (1951) 472.

[33] A.H. Taub, Phy. Rev. 103 (1956) 454.

[34] N. Tomimura, II Nuovo Cimento B 44 (1978) 372.

[35] P. Szekeres, Commun. Math. Phys. 41 (1975) 55.

[36] J.M.M. Senovilla, Phy. Rev. Lett. 64 (1990) 2219.

[37] E. Ruiz and J.M.M. Senovilla, Phy. Rev. D 45 (1990) 1995.

[38] N. Dadhich, R. Tikekar, and L.K. Patel, Curr. Sci. 65 (1993) 694.

[39] R. Bali and A. Tyagi, Astrophys. Space Sc. 173 (1990) 233. 
[40] A. Pradhan, A. K. Yadav and J. P. Singh, Fizika B (Zagreb) 16 (2007) 175 ;

A Pradhan P Singh and A. K. Yadav gr-qc/0705.4411.(2007).

[41] Ya. B. Zeldovich, A.A. Ruzmainkin, and D.D. Sokoloff, Magnetic field in Astrophysics, Gordon and Breach, New Yark (1983).

[42] E.R. Horrison, Phys. Rev. Lett. 30 (1973) 188.

[43] H.P. Robertson and A.G. Walker, Proc. London Math. Soc. 42 (1936) 90

[44] Asseo E and Sol H 1987 Phys. Rep. 6148.

[45] L.P. Hughston and K.C. Jacobs, Astrophys. J. 160, (1970) 147.

[46] J.D. Barrow, Phys. Rev. D 55 (1997) 7451.

[47] Ya. A. Zeldovich, Sov. Astron. 13 (1970) 608.

[48] M.S. Turner and L.M. Widrow, Phys. Rev. D 30 (1988) 2743.

[49] J. Quashnock, A. Loeb, and D.N. Spergel, Astrophys. J. 344 (1989) L49.

[50] A.D. Dolgov, Phys. Rev. D 48 (1993) 2499.

[51] L. Marder, Proc. Roy. Soc. A 246 (1958) 133.

[52] . A. Lichnerowicz, Relativistic Hydrodynamics and Magnetohydrodynamics, W. A. Benzamin. Inc. New York, Amsterdam (1967) p. 93.

[53] J. L. Synge, Relativity: The General Theory, North-Holland Publ., Amsterdam (1960) p. 356.

[54] J. Gunn and B. M. Tinsley, Nature, 257 (1975) 454.

[55] E. J. Wampler and W. L. Burke, New Ideas in Astronomy, eds. F. Bertola, J. W. sulentic, and B. F. Madore, Cambridge University Press (1988) p. 317.

[56] A. D. Sakharov, Doklady Akad. Nauk. SSSR 177 (1968) 70 (translation,Soviet Phys. Doklady, 12 (1968)) 1040.

[57] P. J. E. Peebles and B. Ratra, Astrophys. J. 325 (1988) L17.

[58] J. V. Narlikar, J. -C. Pecker and J. -P. Vigier, J. Astrophys. Astr. 12 (1991) 7.

[59] S. Ray and D. Ray, Astrophys. Space Sci. 203 (1993) 211.

[60] R. N. Tiwari, S. Ray and S. Bhadra, Indian Pure Appl. Math. 31 (2000) 1017.

[61] G.F.R. Ellis, General Relativity and Cosmology,ed R. K. Sachs Charendon Press (1973) p. 117 
[62] S.W. Hawking and G.F.R. Ellis, Large-scale Structure of Space Time, Cambridge University Press, Cambridge (1973) p. 94.

[63] F. C. Santos, M. L. Bedran and V. Soares, Phys. Lett. B 636 (2006) 86.

[64] F. C. Santos, V. Soares and M. L. Bedran, Phys. Lett. B 646 (2007) 215.

[65] E. W. Kolb and M. S. Turner, The Early Universe, Addison-Wesley (1990) p.65.

[66] Y. S. Myung, arXiv:0810.4385.(2008).

[67] R. Maartens, Pramana J. Phys. 55 (4), (2000) 575.

[68] A. K. Yadav and A. Bagora, Fizika B (Zagreb) 18 (2009) 165. 